\title{
Dusty Disks in the Multiple Systems UZ Tau and GG Tau ${ }^{1}$
}

\author{
M. SIMON \\ Astronomy Program, SUNY, Stony Brook, NY 11794, USA \\ S. GUILLOTEAU \\ IRAM, 300 Rue de la Piscine, F-38406 Saint Martin d'Hères, France
}

\begin{abstract}
Interferometric observations of the $2.6 \mathrm{~mm}$ dust thermal emission around the $T$ Tauri triple system UZ Tau show that most of it is equally divided between UZ Tau W (the close binary) and UZ Tau E. The emission is at least partially optically thick at $2.6 \mathrm{~mm}$ which implies an origin in disks of size $\sim 13 \mathrm{AU}$ and mass $\sim 0.024 \mathcal{M}_{\odot}$. The $2.6 \mathrm{~mm}$ emission of the GG Tau system, a hierarchical quadruple, is partially resolved. Strong emission extended over $\sim 3^{\prime \prime} \times 5^{\prime \prime}$ is associated with the close binary GG Tau. Weak emission is detected at GG Tau/c, also a close binary. Evidently extensive dusty disks can survive in the environment of close binaries for at least $\sim 10^{5} \mathrm{y}$, and their structure can vary markedly from system to system.
\end{abstract}

\footnotetext{
${ }^{1}$ Astrophysical Journal Letters, submitted.
} 